\title{
Association between coronary artery calcium score and carotid atherosclerotic disease
}

\author{
QIAN ZHAO $^{1,2}$, XINHUAI WU $^{1}$, JIANMING CAI ${ }^{2}$, XIHAI ZHAO $^{2}$, SHAOHONG ZHAO $^{2}$, \\ LI YANG $^{2}$ and ZULONG CAI ${ }^{2}$ \\ ${ }^{1}$ Department of Radiology, The Beijing Military Region General Hospital of Chinese PLA, Beijing 100700; \\ ${ }^{2}$ Department of Radiology, Chinese PLA General Hospital, Beijing 100853, P.R. China
}

Received January 7, 2013; Accepted April 22, 2013

DOI: $10.3892 / \mathrm{mmr} .2013 .1521$

\begin{abstract}
The aim of the present study was to evaluate the association between coronary calcification and carotid atherosclerosis. Briefly, 123 patients with suspected coronary artery disease were recruited. The coronary artery calcium score (CACS) was measured and multi-contrast carotid magnetic resonance imaging (MRI) was performed. Parameters of the carotid morphological measurements were determined and the presence or absence of carotid plaque tissue compositions was recorded for each subject, as well as plaque surface disruption. Two reviewers blind to CACS status interpreted the MRIs. All the subjects were divided into two groups, high- and low-CACS and the carotid morphological measurements in the CACS groups and categories were compared using independent t-tests and one way ANOVA, respectively. CACS was found to exhibit a moderate correlation with carotid wall area (WA; $\mathrm{r}=0.521$ ) and mean wall thickness (MWT; $r=0.556$ ) and a weak correlation with total vessel area (TVA; $r=0.215)$ and normalized wall index $(r=0.377)$. No significant correlation between CACS and carotid lumen area was identified. Compared with low-CACS, the high-CACS group revealed increased carotid WA, TVA and MWT. In the entire cohort, carotid plaque burden revealed an increasing trend with an increasing CACS. CACS and presence of carotid calcification and lipid-rich necrotic core (LRNC) demonstrated a significant correlation. No significant correlation was observed between CACS with carotid intraplaque hemorrhage (IPH) and surface disruption. CACS was found to moderately correlate with carotid calcification and LRNC volume and weakly correlate with carotid IPH volume. Results of the present study indicated that CACS significantly correlates with carotid atherosclerotic plaque burden and the presence of tissue compositions.
\end{abstract}

Correspondence to: Dr Zulong Cai, Department of Radiology, Chinese PLA General Hospital, 28 Fuxing Road, Beijing 100853, P.R. China

E-mail: zulongcai@163.com

Key words: coronary artery, carotid artery, atherosclerosis, computed tomography, magnetic resonance imaging

\section{Introduction}

Atherosclerotic disease has been demonstrated to be associated with cardiovascular events, including myocardial infarction, transient ischemia attack (TIA) and ischemic stroke $(1,2)$. Atherosclerosis is a systemic arterial disease which frequently involves multiple vascular beds $(3,4)$. Etiologically, cases of atherosclerosis occurring in different arterial vascular beds may share the same set of traditional risk factors (5). Therefore, individuals who develop atherosclerotic disease in one vascular bed may have concomitant atherosclerosis in other vasculatures. Therefore, atherosclerotic incidence in one arterial circulation may be a predictor of atherosclerosis in other arterial territories.

A number of previous studies demonstrated a significant correlation between coronary and carotid atherosclerosis (6-8). Coronary artery calcium score (CACS) was demonstrated to represent an effective predictor of carotid atherosclerotic disease as measured by ultrasound intima-media thickness (IMT) $(9,10)$. In addition to measurement of plaque burden, more recently, characterization of carotid compositional features, including lipid-rich necrotic core (LRNC), intraplaque hemorrhage (IPH) and surface disruption, as determined by high-resolution, multi-contrast magnetic resonance imaging (MRI), have been hypothesized to be clinically significant due to their associations with neurovascular events (11-13). However, the predictive value of CACS for carotid compositional features remains unknown.

The present study aimed to determine the association between CACS and carotid atherosclerotic disease and the predictive value of CACS for plaque burden and compositional features in carotid arteries as determined by high-resolution, multi-contrast MRI.

\section{Materials and methods}

Study population. Individuals who were suspected to suffer from coronary artery disease (CHD) with chest pain were recruited. The study was conducted in accordance with the declaration of Helsinki and the study protocol was approved by the Ethics Committee and the Institutional Review Board of the Chinese PLA General Hospital. All patients provided written informed consent. The exclusion criteria included 
contraindications to MR scan. The subjects underwent CT scan for CACS exam and carotid high-resolution, multi-contrast, bilateral carotid MR imaging within 2 weeks.

CACS data acquisition and image processing. A low-dose CACS protocol was performed to capture coronary pre-contrast images for CACS measurement using a Dual Source CT scanner (Dual Source CT; Siemens, Forchheim, Germany). The parameters of the low-dose CACS protocol were as follows: $120 \mathrm{kV} ; 80 \mathrm{~mA} / \mathrm{rot}$; pitch, 0.2-2.0; rotation time, $0.33 \mathrm{sec} / \mathrm{rot}$; and slice thickness, $3.0 \mathrm{~mm}$. CACS was computed using commercial software (Syngo MultiModality Workplace; Siemens) and Agatston algorithm (14) by an experienced radiologist.

Carotid MR imaging. Carotid MR imaging was performed using a dedicated, phased-array, surface coil and a 3.0T scanner (GE Signa Excite; GE Healthcare, Waukesha, WI, USA). Cross-sectional images of the carotid arteries were captured using four contrast-weighted images: T1-weighted (T1W), proton density-weighted (PDW), T2-weighted (T2W) and three-dimensional (3D) time of flight (TOF). Parameters for the imaging sequences were as follows: T1W: quadruple inversion-recovery (QIR), black-blood, 2D fast spin-echo, TR/TE 800/8.8 msec; PDW and T2W: double echo, TR=3,000 msec, TE $13.1 \mathrm{msec}$ for PDW and $56.9 \mathrm{msec}$ for T2W; and 3D TOF: TR/TE 29/2.1 msec, flip angle $20^{\circ}$. Images were captured under a field of view of $14 \mathrm{~cm}$ and matrix size $256 \times 256$ for an in-plane acquisition resolution of $0.55 \times 0.55 \mathrm{~mm}^{2}$. Axial images of the bilateral carotid arteries were captured with a 2-mm slice thickness over a longitudinal coverage of $32 \mathrm{~mm}$.

Interpretation of carotid MR images. The carotid MR images were interpreted by two trained reviewers via consensus opinion blinded to clinical information and CACS results. For MR image review, CASCADE image analysis software (15) was used to outline the lumen and outer wall boundaries. The lumen area (LA), wall area (WA), total vessel area (TVA), mean wall thickness (MWT) and normalized wall index $(\mathrm{NWI}=\mathrm{WA} / \mathrm{TVA})$ were measured for each axial location. Carotid artery was divided into three segments: internal carotid artery (ICA), defined as the segment above the bifurcation; bulb, defined as the segment from the bifurcation to $4 \mathrm{~cm}$ below; and common carotid artery (CCA), defined as the segment below the bulb. The mean values of LA, WA, TVA, MWT and NWI of bilateral arteries for each subject and each carotid segment were calculated. The presence or absence of carotid plaque compositions, including calcification, LRNC, IPH and surface disruption were also identified and recorded for each carotid segment and each subject, respectively, using criteria described previously (16).

Statistical analysis. The correlation of CACS with carotid morphological measurements, including LA, WA, TVA, MWT and NWI and the presence or absence of plaque compositions, such as calcification, LRNC and IPH were evaluated. The correlation between CACS and the volumes of carotid calcification, LRNC and IPH in subjects was also determined. CACS was divided into three categories: $\mathrm{CACS}=0,=1-399$ and
Table I. Demographic characteristics of the study population $(n=123)$.

\begin{tabular}{lcc}
\hline Characteristics & $\begin{array}{c}\text { Mean } \pm \text { SD } \\
\text { or } \mathrm{n}(\%)\end{array}$ & $\begin{array}{c}\text { Range } \\
\text { (where applicable) }\end{array}$ \\
\hline Gender, male & $96(78)$ & - \\
Age, years & $58.0 \pm 9.5$ & $44-84$ \\
Height, $\mathrm{cm}$ & $170.1 \pm 6.9$ & $152-188$ \\
Weight, $\mathrm{kg}$ & $75.4 \pm 10.8$ & $50-100$ \\
BMI & $26.0 \pm 2.9$ & $19.0-33.8$ \\
TC, $\mathrm{mg} / \mathrm{dl}$ & $192.3 \pm 41.9$ & $116.0-382.8$ \\
HDL, mg/dl & $45.8 \pm 14.3$ & $18.2-144.2$ \\
LDL, mg/dl & $104.9 \pm 29.2$ & $35.2-191.0$ \\
SBP, mmHg & $148.6 \pm 26.4$ & $100-240$ \\
DBP, mmHg & $91.5 \pm 16.7$ & $62-130$ \\
Hypertension & $72(58.5)$ & - \\
Diabetes & $30(24.4)$ & - \\
Smoking & $67(54.5)$ & -
\end{tabular}

BMI, body mass index; TC, total cholesterol; HDL, high-density cholesterol; LDL, low-density cholesterol; SBP, systolic blood pressure; DBP, diastolic blood pressure.

$>400$ (17). Morphological measurements and the prevalence of plaque compositions in each CACS category were determined. Univariate regression was used to determine the correlation between CACS and carotid plaque burden and compositional features. Multivariate regression was also conducted to evaluate the correlation between CACS and carotid plaque burden and compositional features following adjustment for confounding factors. The odds ratios (OR) and corresponding 95\% confidential interval (CI) of CACS were calculated to predict the presence of carotid calcification, LRNC and IPH with an increment of $100 . \mathrm{P}<0.05$ was considered to indicate a statistically significant difference. Analyses were performed with SPSS software for windows (version 12.0; SPSS, Inc., Chicago, IL, USA).

\section{Results}

Patient characteristics. Between May 2008 and February 2009, 128 patients with suspected CAD were recruited. Of 128 patients, 5 were excluded from the final analysis due to poor MR image quality. Of the remaining 123 patients, 96 were male (mean age, 58.0 \pm 9.5 years), 60 had carotid calcification, 79 had LRNC, 12 had IPH and 4 had surface disruption. The demographic characteristics for the patients are provided in Table I. Surface disruption was not included in the statistical analysis due to a limited number of cases.

Correlation between CACS and carotid morphology. CACS was found to significantly correlate with WA, TVA, MWT and NWI for carotid artery ( $r=0.208-0.529$; $\mathrm{P}<0.05$; Fig. 1). Similarly, significant correlations between CACS with WA, TVA, MWT and NWI for ICA, bulb and CCA were also found. 
Table II. Correlation between carotid plaque compositions and CACS.

\begin{tabular}{|c|c|c|c|c|c|}
\hline \multirow[b]{2}{*}{ Parameter } & \multicolumn{3}{|c|}{ CACS categories } & \multirow[b]{2}{*}{$\mathrm{r}$} & \multirow[b]{2}{*}{ P-value } \\
\hline & $0(n=35)$ & $1-399(n=66)$ & $>400(\mathrm{n}=22)$ & & \\
\hline \multicolumn{6}{|l|}{ Carotid artery } \\
\hline Calcification & 11.4 & 60.6 & 72.7 & 0.479 & $<0.001$ \\
\hline LRNC & 25.7 & 78.8 & 81.8 & 0.394 & $<0.001$ \\
\hline IPH & 0 & 13.6 & 13.6 & 0.208 & 0.021 \\
\hline \multicolumn{6}{|l|}{ ICA } \\
\hline Calcification & 5.7 & 33.8 & 54.5 & 0.426 & $<0.001$ \\
\hline LRNC & 5.7 & 33.8 & 40.9 & 0.237 & 0.009 \\
\hline IPH & 0 & 6.2 & 9.1 & 0.138 & 0.130 \\
\hline \multicolumn{6}{|l|}{ Bulb } \\
\hline Calcification & 8.6 & 48.5 & 63.6 & 0.466 & $<0.001$ \\
\hline LRNC & 17.1 & 66.7 & 68.2 & 0.368 & $<0.001$ \\
\hline IPH & 0 & 7.6 & 9.1 & 0.205 & 0.023 \\
\hline \multicolumn{6}{|l|}{ CCA } \\
\hline Calcification & 0 & 25.8 & 68.2 & 0.510 & $<0.001$ \\
\hline LRNC & 22.9 & 59.1 & 59.1 & 0.244 & 0.007 \\
\hline IPH & 0 & 3.0 & 0 & 0.044 & 0.629 \\
\hline
\end{tabular}

CACS, coronary artery calcium score; LRNC, lipid-rich necrotic core; IPH, intraplaque hemorrhage; ICA, internal carotid artery, CCA, common carotid artery.

Table III. Correlation between CACS and carotid plaque composition prior to and following adjustment for confounding factors.

\begin{tabular}{|c|c|c|c|c|}
\hline \multirow[b]{2}{*}{ Parameters } & \multicolumn{2}{|c|}{ CACS prior to adjustment } & \multicolumn{2}{|c|}{ CACS following adjustment } \\
\hline & OR & P-value & OR & P-value \\
\hline Calcification & $1.320(1.110-1.570)$ & 0.002 & $1.369(1.153-1.624)$ & $<0.001$ \\
\hline LRNC & $1.209(1.019-1.435)$ & 0.030 & $1.148(0.962-1.369)$ & 0.126 \\
\hline IPH & $1.060(0.978-1.148)$ & 0.156 & $1.032(0.9421-1.132)$ & 0.498 \\
\hline Surface disruption & $0.925(0.636-1.346)$ & 0.684 & $0.206(0.022-1.939)$ & 0.167 \\
\hline
\end{tabular}

P-value was calculated using univariate and multivariate regression models with 100 increment for CACS prior to and following adjustment for confounding factors, including age, gender, BMI, hypertension, hyperlipidemia, diabetes and smoking. LRNC, lipid-rich necrotic core; IPH, intraplaque hemorrhage; CACS, CACS, coronary artery calcium score; OR, odds ratio.

In addition, CACS was found to positively correlate with LA for carotid artery and CCA, but not for ICA and bulb. Of note, CACS was observed to correlate more closely with NWI of ICA and bulb than that of CCA.

Following adjustment for confounding factors, including age, gender, BMI, hypertension, hyperlipidemia, diabetes and smoking, CACS remained significantly correlated with MWT for carotid artery and all its segments $(\mathrm{P}<0.01)$. Similarly, a significant correlation of CACS with NWI for carotid artery and segments ICA and bulb were also found. CACS positively correlated with NWI for CCA $(\beta=0.238 ; \mathrm{P}=0.028)$ prior to adjustment for confounding factors, but not following adjustment $(\beta=0.178 ; \mathrm{P}=0.115)$. Of note, $\mathrm{CACS}$ was found to correlate more closely with NWI of ICA and bulb than that of CCA.
Correlation between CACS and carotid plaque compositions. A significant moderate correlation was observed between CACS and the presence of calcification for carotid artery and its segments (ICA, bulb and CCA; $r=0.426-0.510 ; \mathrm{P}<0.05$; Table II). In addition, a weak correlation between CACS and the presence of LRNC in carotid artery and its three segments was observed $(r=0.237-0.394$; $\mathrm{P}<0.05)$. For IPH, a significant weak correlation with CACS was found in carotid artery $(\mathrm{r}=0.208 ; \mathrm{P}=0.021)$ and bulb $(\mathrm{r}=0.205 ; \mathrm{P}=0.023)$. However, no arteries in patients with $\mathrm{CACS}=0$ developed IPH. In patients with carotid calcification $(n=60)$, a marked correlation was found between carotid calcification volume and CACS ( $\mathrm{r}=0.747$; $\mathrm{P}<0.001$; Fig. 2). For arteries with LRNC $(n=79)$, CACS did not correlate with LRNC volume $(r=0.183$; 

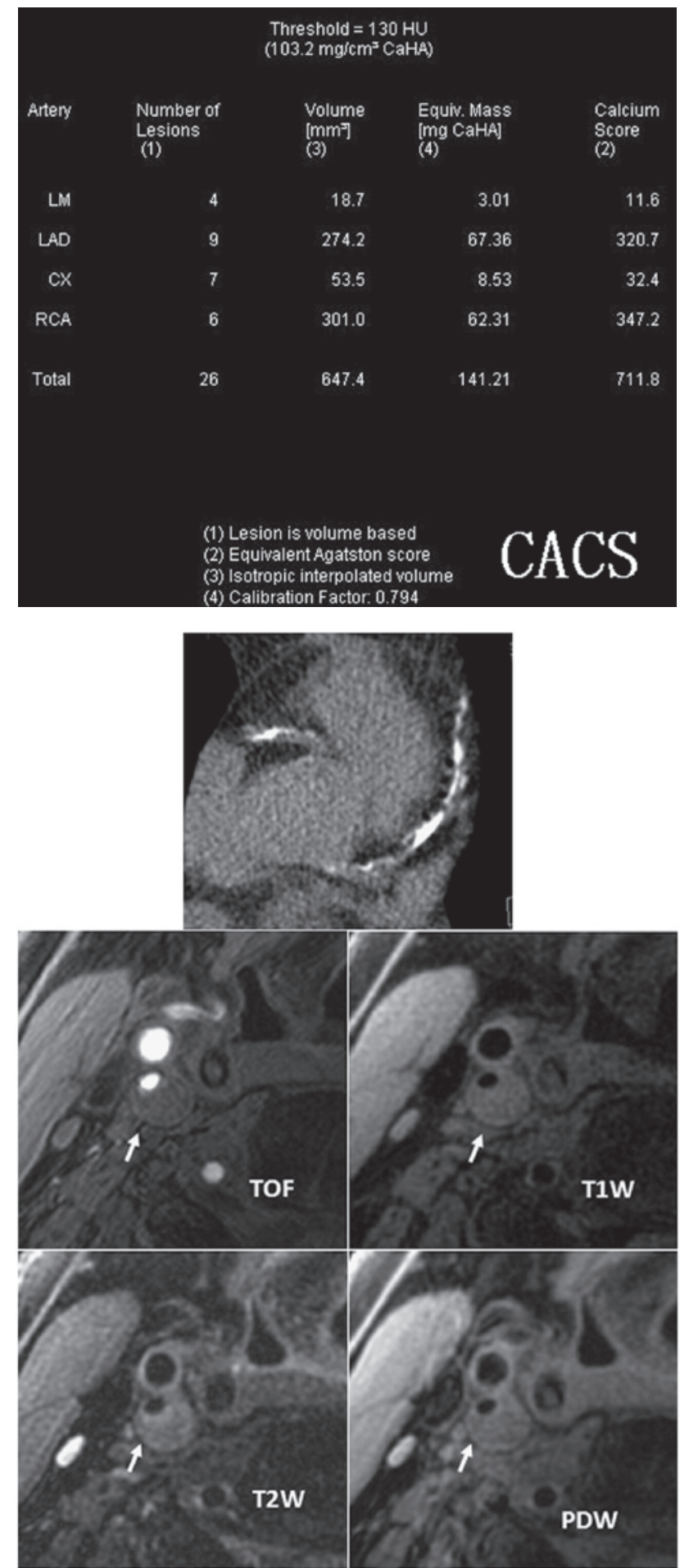

Figure 1. High CACS $(=711.8)$ in coronary artery CT images and increased MWA $\left(=44.66 \mathrm{~mm}^{2}\right)$ and MWT on the right internal carotid artery MRI in the same patient. CACS, coronary artery calcium score; MWA, mean wall areal; MWT, mean wall thickness; MRI, magnetic resonance image.

$\mathrm{P}=0.107)$. Similarly, there was no significant correlation between CACS and IPH volume $(\mathrm{r}=-0.055 ; \mathrm{P}=0.865)$ in arteries with IPH $(\mathrm{n}=12)$.

In addition, in CACS predicting the presence of carotid calcification, LRNC and IPH, OR was 1.32 (95\% CI, 1.11-1.57; $\mathrm{P}=0.002), 1.21(95 \% \mathrm{CI}, 1.02-1.44 ; \mathrm{P}=0.029)$ and $1.06(95 \%$ CI, 0.98-1.15; $\mathrm{P}=0.156$ ), respectively. However, following
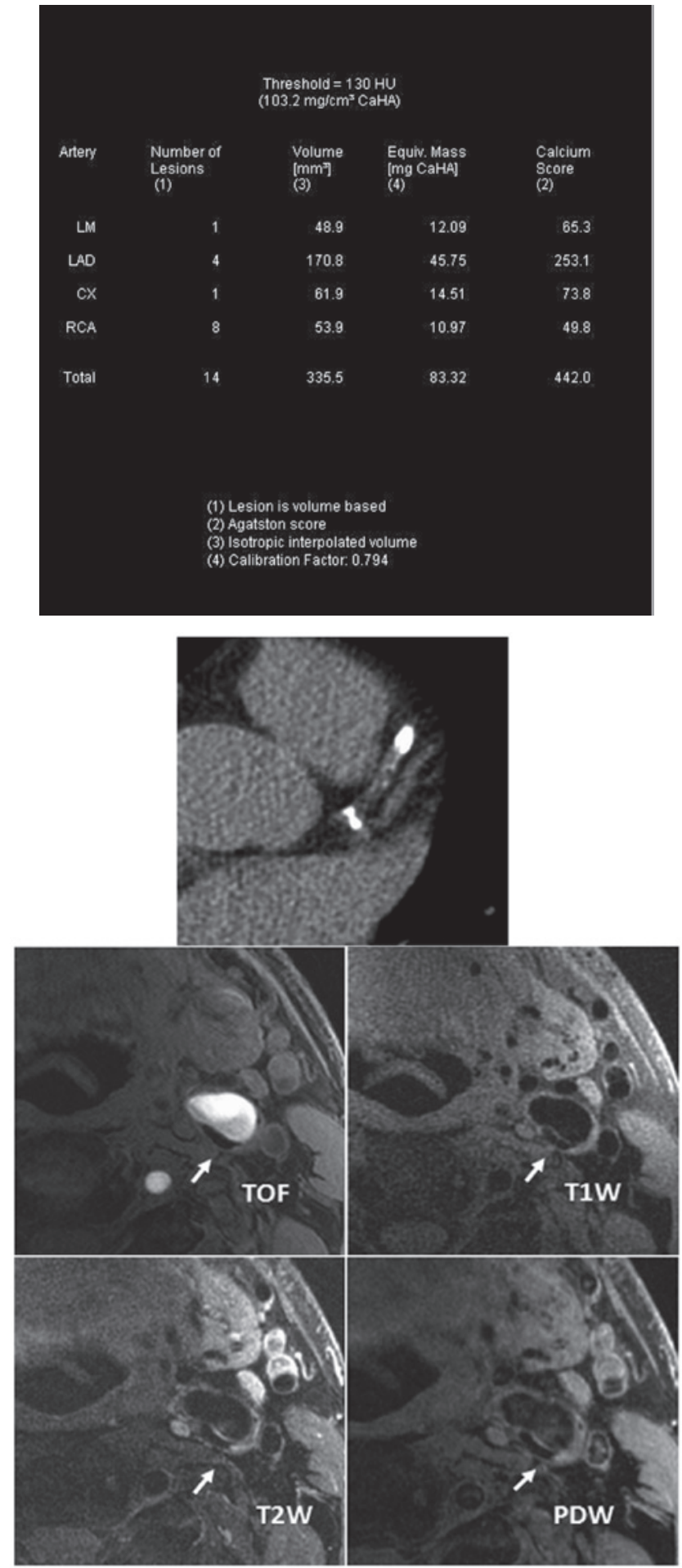

Figure 2. Male patient, 76 years old. High CACS (=422.0) was revealed in coronary artery $\mathrm{CT}$ images and increased calcification on the left internal carotid artery MRIs in the same patient. CACS, coronary artery calcium score; MRIs, magnetic resonance images.

adjustment for confounding factors, OR was $1.148(\mathrm{P}>0.05)$ in predicting presence of carotid LRNC (Table III).

\section{Discussion}

In the present study, the association of CACS with carotid atherosclerosis and the predictive value of CACS for carotid 
atherosclerotic disease was determined. Results indicate that CACS is significantly associated with carotid plaque burden and compositional features. In addition, CACS was found to be an effective predictor of the presence of carotid calcification and LRNC. These observations are consistent with the hypothesis that atherosclerosis is a systemic disease that frequently involves multiple vascular territories. In addition, results indicate that CACS may be a useful predictor of carotid plaque burden and compositional features, particularly calcification and LRNC.

The identification of a correlation between CACS and carotid plaque burden is consistent with previous studies $(6,10,18)$. In a study using IMT to measure carotid plaque burden by B-mode ultrasound, El-Saed et al (10) demonstrated that CACS significantly correlates with carotid IMT $(r=0.47$, $\mathrm{P}<0.001)$. Similar results have also been reported in additional studies $(7,19)$. In the present study, MWT and NWI were used as a surrogate of IMT for plaque burden measurement due to consistencies between IMT and MWT (20). In addition, the correlation between CACS and various segments of carotid artery was investigated and ICA and bulb, particularly bulb, were found to reveal a slightly stronger correlation with CACS than CCA. This correlation difference may be explained by the high prevalence of atherosclerotic plaques and the complex hemodynamic properties in the bulb region (21). Since CACS has been demonstrated to be a powerful measure of coronary disease severity, current observations of a positive correlation between CACS and carotid plaque burden indicate that the severity of atherosclerotic disease in coronary and carotid arteries may be parallel. In their study, Bauer et al reported a weak correlation between CACS and IMT $(r=0.26 ; \mathrm{P}<0.0001)$ among 1,620 males without CAD and stroke, aged 45-75 years, indicating that CACS is not sufficient to predict carotid IMT. However, the study population examined by Bauer et al was different to that of the present study, as all participants were male, and had no CAD or history of stroke (22).

In the present study, a significant correlation between CACS and prevalence and volume of carotid plaque calcification was identified. Consistent with a study by Odink et al (18), CACS was found to positively correlate with carotid calcification volume. In addition, results of the present study are also supported by a study by Allison et al (23), in which 650 asymptomatic patients receiving whole-body electron beam computed tomography scanning were analyzed to assess the carotid, coronary, proximal and distal aorta and iliac vessels for atherosclerotic calcification. The authors of that study found that age and hypertension represented the dominant risk factors for systemic calcified atherosclerosis and a positive correlation existed between the coronary and carotid beds (0.28-0.29). Etiologically, atherosclerosis occurring in various arterial vascular beds may share the same set of traditional risk factors. Schlieper et al (24) reported that age, male gender, dialysis vintage, smoking, calcium-phosphate product and high-sensitivity CRP are independent risk factors for cardiovascular calcifications, including coronary and carotid calcification. Observations of the present study confirm the presence of significant correlations and risk factor associations for calcified atherosclerosis in various vascular beds. However, carotid calcification plays a controversial role in plaque vulnerability. Although the possible contribution of calcification to plaque stabilization requires additional clarification, a number of previous studies have reported that calcification is markedly associated with plaque rupture and subsequent thrombosis. Demer et al (25) found that calcified human vessels were less distensible in vivo and ex vivo compared with non-calcified vessels. Studies performed by Richardson et al (26), based on computer modeling, as well as Lee et al (27), with use of computer-assisted analysis of tensile stress on excised plaques, demonstrated that sites prone to rupture were often associated with the greatest tensile stress in the vessel wall, which occurs at the interphases between two tissues with differing elastic properties.

To the best of our knowledge, no studies have investigated the link between CACS and carotid LRNC and IPH. In the current study, a positive correlation was identified between CACS and the volume of carotid LRNC and presence of IPH. In addition, our results indicate that CACS correlates with carotid plaque stability.

It is evident from a series of studies that carotid plaque complexity and composition may be associated with plaque stability. Previous pathological studies established that a large LRNC or IPH is an important feature of vulnerable atherosclerotic plaque. The size of carotid LRNC and presence of IPH were demonstrated to be high-risk features due to their correlation with neurovascular events. Cappendijk et al (28) reported that symptomatic patients exhibited wider ranges in LRNC scores than asymptomatic individuals. Altaf et al (29) prospectively studied symptomatic patients with mild to moderate carotid stenosis and found that patients with carotid IPH were prone to recurrent neurological events, including TIA and stroke. In addition, a study by the same research group identified a significant and marked association between recurrent clinical ischemia and intraplaque hemorrhage in patients with symptomatic high-grade carotid disease (30).

In the present study, the prevalence and volume of carotid LRNC and presence of IPH revealed an increasing trend with CACS, indicating that CACS may be associated with carotid plaque vulnerability.

This study has concentrated on a specific time point, and mainly analyzed the correlation between CACS and carotid atherosclerosis, however, the predictive value of CACS to carotid atherosclerosis requires a larger prospective study to confirm. Although CACS was revealed to predict carotid atherosclerosis, additional studies must be performed. In addition, only four patients developed carotid surface disruption. In future studies the correlation between CACS and carotid surface disruption must be determined using a larger study population.

\section{References}

1. Dalager-Pedersen S, Ravn HB and Falk E: Atherosclerosis and acute coronary events. Am J Cardiol 82: 37-40, 1998.

2. Iannuzzi A, Wilcosky T, Mercuri M, et al: Ultrasonographic correlates of carotid atherosclerosis in transient ischemic attack and stroke. Stroke 26: 614-619, 1995.

3. CAPRIE Steering Committee: A randomised, blinded, trial of clopidogrel versus aspirin in patients at risk of ischaemic events (CAPRIE). CAPRIE Steering Committee. Lancet 348: 1329-1339, 1996.

4. Kannel WB and Wolf PA: Peripheral and cerebral atherothrombosis and cardiovascular events in different vascular territories: insights from the Framingham Study. Curr Atheroscler Rep 8: 317-323, 2006. 
5. Meco JF, Pintó X, Escribà JM, et al: Cardiovascular risk factors associated with clinically isolated and diffuse atherosclerosis in Spanish patients with coronary artery disease. Eur J Clin Invest 28: 643-650, 1998

6. Rampersaud E, Bielak LF, Parsa A, et al: The association of coronary artery calcification and carotid artery intima-media thickness with distinct, traditional coronary artery disease risk factors in asymptomatic adults. Am J Epidemiol 168: 1016-1023, 2008.

7. Taylor AJ, Bindeman J, Le TP, et al: Progression of calcified coronary atherosclerosis: relationship to coronary risk factors and carotid intima-media thickness. Atherosclerosis 197: 339-345, 2008

8. Underhill HR, Yuan C, Terry JG, et al: Differences in carotid arterial morphology and composition between individuals with and without obstructive coronary artery disease: a cardiovascular magnetic resonance study. J Cardiovasc Magn Reson 10: 31 , 2008.

9. Manolio TA, Arnold AM, Post W, et al: Ethnic differences in the relationship of carotid atherosclerosis to coronary calcification the Multi-Ethnic Study of Atherosclerosis. Atherosclerosis 197: 132-138, 2008.

10. El-Saed A, Sekikawa A, Edmundowicz D, et al: Coronary calcification is more predictive of carotid intimal medial thickness in black compared to white middle aged men. Atherosclerosis 196 913-918, 2008.

11. Cai J, Hatsukami TS, Ferguson MS, et al: In vivo quantitative measurement of intact fibrous cap and lipid-rich necrotic core size in atherosclerotic carotid plaque: comparison of high-resolution, contrast-enhanced magnetic resonance imaging and histology. Circulation 112: 3437-3444, 2005.

12. Chu BC, Kampschulte A, Ferguson MS, et al: Hemorrhage in the atherosclerotic carotid plaque: a high-resolution MRI study. Stroke 35: 1079-1084, 2004.

13. Chu B, Phan BA, Balu N, et al: Reproducibility of carotid atherosclerotic lesion type characterization using high resolution multicontrast weighted cardiovascular magnetic resonance. J Cardiovasc Magn Reson 8: 793-799, 2006.

14. Agatston AS, Janowitz WR, Hildner FJ, et al: Quantification of coronary artery calcium using ultrafast computed tomography. J Am Coll Cardiol 15: 827-832, 1990

15. Kerwin W, Xu D, Liu F, et al: Magnetic resonance imaging of carotid atherosclerosis: plaque analysis. Top Magn Reson Imaging 18: 371-378, 2007.

16. Saam T, Ferguson MS, Yarnykh VL, et al: Quantitative evaluation of carotid plaque composition by in vivo MRI. Arterioscler Thromb Vasc Biol 25: 234-239, 2005.

17. Schenker MP, Dorbala S, Hong EC, et al: Interrelation of coronary calcification, myocardial ischemia and outcomes in patients with intermediate likelihood of coronary artery disease: a combined positron emission tomography/computed tomography study. Circulation 117: 1693-1700, 2008.
18. Odink AE, van der Lugt A, Hofman A, et al: Association between calcification in the coronary arteries, aortic arch and carotid arteries: the Rotterdam study. Atherosclerosis 193: 408-413, 2007.

19. Newman AB, Naydeck BL, Ives DG, et al: Coronary artery calcium, carotid artery wall thickness, and cardiovascular disease outcomes in adults 70 to 99 years old. Am J Cardiol 101: $186-192,2008$

20. Underhill HR, Kerwin WS, Hatsukami TS and Yuan C: Automated measurement of mean wall thickness in the common carotid artery by MRI: a comparison to intima-media thickness by B-mode ultrasound. J Magn Reson Imaging 24: 379-387, 2006.

21. Salzar RS, Thubrikar MJ and Eppink RT: Pressure-induced mechanical stress in the carotid artery bifurcation: a possible correlation to atherosclerosis. J Biomech 28: 1333-1340, 1995.

22. Bauer M, Mohlenkamp S, Lehmann N, et al: The effect of age and risk factors on coronary and carotid artery atherosclerotic burden in males - results of the Heinz Nixdorf recall study. Atherosclerosis 205: 595-602, 2009.

23. Allison MA, Criqui MH and Wright CM: Patterns and risk factors for systemic calcified atherosclerosis. Arterioscler Thromb Vasc Biol 24: 331-336, 2004.

24. Schlieper G, Brandenburg V, Djuric Z, et al: Risk factors for cardiovascular calcifications in non-diabetic Caucasian haemodialysis patients. Kidney Blood Press Res 32: 161-168, 2009.

25. Demer LL: Effect of calcification on in vivo mechanical response of rabbit arteries to balloon dilation. Circulation 83: 2083-2093, 1991.

26. Richardson PD, Davies MJ and Born GV: Influence of plaque configuration and stress distribution on fissuring of coronary atherosclerotic plaques. Lancet 2: 941-944, 1989.

27. Lee RT, Schoen FJ, Loree HM, et al: Circumferential stress and matrix metalloproteinase 1 in human coronary atherosclerosis. Implications for plaque rupture. Arterioscler Thromb Vasc Biol 16: 1070-1073, 1996.

28. Cappendijk VC, Kessels AG, Heenemao S, et al: Comparison of lipid-rich necrotic core size in symptomatic and asymptomatic carotid atherosclerotic plaque: initial results. J Magn Reson Imaging 27: 1356-1361, 2008.

29. Altaf N, Daniels L, Morgan PS, et al: Detection of intraplaque hemorrhage by magnetic resonance imaging in symptomatic patients with mild to moderate carotid stenosis predicts recurrent neurological events. J Vasc Surg 47: 337-342, 2008.

30. Altaf N, MacSweeney ST, Gladman J and Auer DP: Carotid intraplaque hemorrhage predicts recurrent symptoms in patients with high-grade carotid stenosis. Stroke 38: 1633-1635, 2007. 